\title{
Vólvulo yeyunal incompleto por linfangioma quístico mesentérico
}

\section{Partial jejunal volvulus due to a mesenteric cystic lymphangioma}

\author{
S. Olivera' ${ }^{1}$, B. De Escalante ${ }^{2}$, A. Morandeira ${ }^{3}$, P. Val-Carreres ${ }^{3}$, M.C. Castillo ${ }^{4}$, T. Castiella ${ }^{5}$
}

\section{RESUMEN}

Los quistes mesentéricos son tumoraciones benignas poco frecuentes entre las que se encuentran los linfangiomas. Su presentación clínica es variable y pueden producir síntomas agudos debido a complicaciones. Se diagnostican sobre todo en edad pediátrica y su pronóstico, tras exéresis completa, suele ser excelente. Presentamos el caso de una paciente de 15 años con dolor abdominal postprandial y palpación de masa blanda en hipogastrio. Los estudios radiológicos mostraron una gran masa polilobulada de contenido quístico que englobaba un asa de yeyuno con volvulación incompleta. El tratamiento fue la exéresis del quiste y del asa de yeyuno y el diagnóstico anatomopatológico fue de linfangioma quístico mesentérico. La paciente está asintomática después de más de 3 años de la intervención.

Palabras clave. Linfangioma. Quiste mesentérico. Vólvulo yeyunal.

\begin{abstract}
Mesenteric cysts are unusual benign tumours that include lymphangioma. Their clinical presentation is variable and acute symptoms can be produced due to complications. This tumour appears especially in childhood, and its prognosis after surgical removal is excellent. We present the case of a 15 year old female patient with symptoms of postprandial abdominal pain and palpation of smooth mass in hypogastrium. Radiological studies showed a big polilobular mass of cystic substance that included a portion of jejune with incomplete volvulus. The treatment was the removal of the cyst and a jejunal portion and the pathological diagnosis was mesenteric cyst lymphangioma. The patient is asymptomatic more than three years after the intervention.
\end{abstract}

Key words. Lymphangioma. Mesenteric cyst. Jejunal volvulus.
1. Servicio de Medicina Interna. Hospital de Denia. Alicante.

2. Servicio de Medicina Interna. Hospital Clínico Universitario "Lozano Blesa». Zaragoza.

3. Servicio de Cirugía General. Hospital Clínico Universitario «Lozano Blesa». Zaragoza.

4. Servicio de Radiodiagnóstico. Hospital Clínico Universitario "Lozano Blesa». Zaragoza.

5. Servicio de Anatomía Patológica. Hospital Clínico Universitario "Lozano Blesa». Zaragoza.

\section{Correspondencia}

Susana Olivera González

Servicio de Medicina Interna

Hospital de Denia. Alicante

E-mail: susana.olivera@yahoo.es

Recepción: 13 de septiembre de 2010

Aceptación provisional: 18 de octubre de 2010

Aceptación definitiva: 25 de octubre de 2010 


\section{INTRODUCCIÓN}

El linfangioma abdominal es una rara tumoración benigna que se incluye entre los quistes mesentéricos. Se trata de tumores de etiología desconocida y que se ven más frecuentemente en pacientes pediátri$\cos ^{1}$. La presentación clínica es muy variable, siendo con frecuencia asintomáticos ${ }^{2}$, lo que favorece el retraso en el diagnóstico, pero pueden cursar con dolor abdominal en más del $80 \%$ de los casos ${ }^{3}$, náuseas, vómitos o con clínica de abdomen agudo debido a diferentes complicaciones. En muchas ocasiones se presentan como masa palpable dolorosa, al igual que en el caso que describimos.

\section{CASO CLÍNICO}

Se trata de una paciente de 15 años de edad, sin antecedentes patológicos reseñables. Presentaba dolor abdominal de 4 años de evolución de tipo cólico, de duración variable y predominio postprandial, localizado en hipogastrio y mesogastrio con pérdida de $5 \mathrm{~kg}$ de peso debido a la disminución voluntaria de la ingesta por el dolor. Refería vómitos ocasionales y estreñimiento. Permaneció afebril en todo momento. Valorada por su médico de familia y por un especialista de Aparato Digestivo, se le realizaron análisis de sangre que fueron normales, fibrogastroscopia y tratamiento erradicador para $H$. pylori por test de ureasa en aliento positivo, que no mejoró la sintomatología. Fue remitida a Psiquiatría para descartar un trastorno del comportamiento alimentario.

A la exploración física la paciente presentaba delgadez (IMC 18) y dolor a la palpación en hipogastrio con sensación de masa blanda en zona infraumbilical. Todos los análisis de sangre practicados, incluidos marcadores tumorales, fueron normales. En la ecografía de abdomen se apreció una masa en hipogastrio de al menos $10 \mathrm{~cm}$ de diámetro, polilobulada, con contenido líquido denso en las de zonas de mayor tamaño. Tras la exploración ginecológica se descartó que la masa dependiera de aparato genital. En la TC de abdomen se observó malposición de asas proximales de intestino delgado y, a nivel pélvico, imagen horizontal en "reloj de arena» de contenido homogéneo quístico, sin pared definida, que no era realzada por el contraste (Fig. 1).

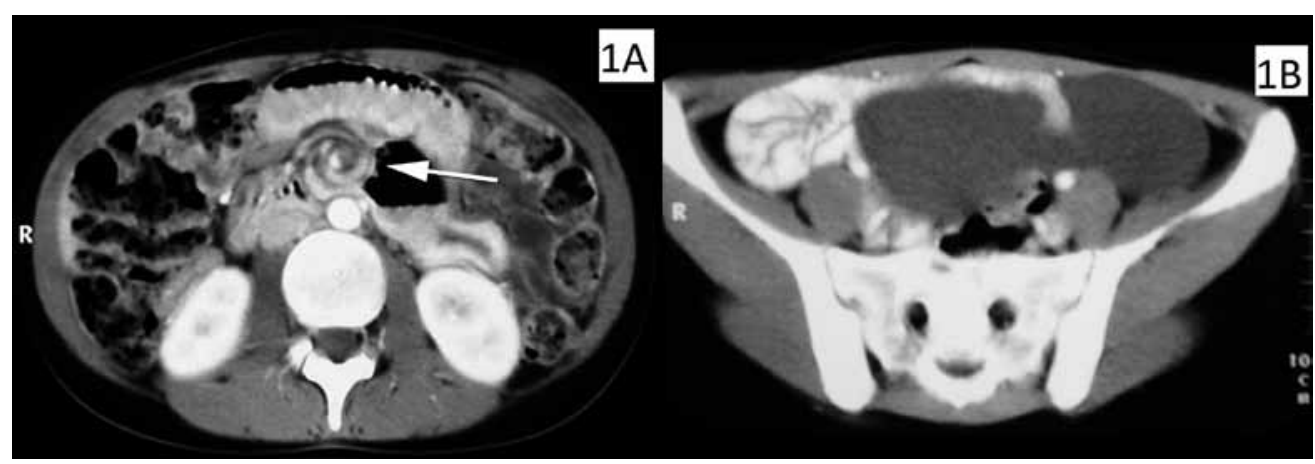

Figura 1. TC abdominal. 1a) Malposición de asas de intestino delgado. La flecha señala la disposición en espiral del mesenterio. 1b) Imagen quística pélvica en «reloj de arena» sin realce de contraste.

El diagnóstico radiológico fue de probable quiste mesentérico, con malposición de asas intestinales. Se realizó tránsito intestinal que mostró imagen en espiral de volvulación incompleta de primeras porciones yeyunales con malposición y desplazamiento de yeyuno e íleon hacia vacío derecho. Ese mismo día la paciente presentó intenso dolor abdominal con náuseas y vómitos, ausencia de emisión de heces y gases y distensión abdominal, que mejoró con dieta absoluta, rehidratación y analgesia potente. La paciente fue intervenida quirúrgicamente y se le practicó laparotomía media supra e infraumbilical, encontrando una gran tumoración de unos $20 \mathrm{~cm}$ de diámetro, de contenido líquido, que nacía del meso intestinal, englobaba un asa de yeyuno y deformaba la situación habitual del intestino. Se practicó quistectomía y resección del asa yeyunal englobada (Fig. 2). El informe anatomopatológico fue de masa quística adheri- 
da a la serosa del asa intestinal, compuesta por numerosos espacios quísticos, algunos multiloculados, que contenían una sustancia serosa acelular, revestidos por endotelio, originados a partir de vasos linfáticos. El diagnóstico fue de linfangioma quístico mesentérico. El asa yeyunal no mostró alteraciones. El postoperatorio fue normal y la paciente se encuentra asintomática sin recidiva de la tumoración en las ecografías practicadas tras más de 3 años de seguimiento.

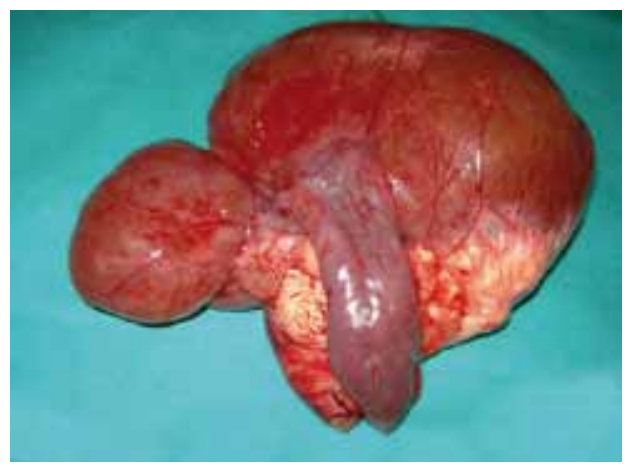

Figura 2. Tumoración quística con asa intestinal englobada.

\section{DISCUSIÓN}

Los linfangiomas pueden localizarse en cualquier parte del cuerpo excepto el cerebro. La mayoría se sitúan en el cuello (75\%), un $20 \%$ en axila y el $4-5 \%$ en mediastino, pulmones, esófago, estómago o intestino ${ }^{4}$. Menos del $1 \%$ afectan al mesenterio, epiplon mayor o retroperitoneo ${ }^{5}$, siendo el mesenterio del intestino delgado el más frecuentemente afectado. Anatomopatológicamente se clasifican en tres tipos: capilar, cavernoso y quístico ${ }^{4,5}$, localizándose estos últimos sobre todo en abdomen y retroperitoneo.

Los linfangiomas quísticos mesentéricos son tumoraciones benignas muy poco frecuentes. Se ha estimado una incidencia de un caso por cada 20.000-250.000 ingresos hospitalarios ${ }^{4}$. Ocurren a cualquier edad, pero la mayoría se presentan en edad pediátrica ${ }^{6}$ y existe un ligero predominio en el sexo femenino ${ }^{4,7}$. Su etiología es desconocida aunque una de las teorías más aceptadas es la del origen embrionario, asociada con anomalías en el desarrollo de los vasos linfáticos y apoyada por su elevada incidencia en la infancia: más del $65 \%$ están presentes en el nacimiento y un $60 \%$ se diagnostican antes de los 5 años $^{4}$. Otras posibles etiologías incluyen el sangrado o inflamación de canales linfáticos que producen obstrucción y posterior formación del linfangioma, aunque esta teoría no es aceptada por algunos autores ${ }^{8}$.

Pueden ser únicos o múltiples, uniloculados o multiloculados y los más frecuentes son los únicos y multiloculados ${ }^{7}$. Es necesario realizar el diagnóstico diferencial con diferentes tumoraciones quísticas como las de ovario, páncreas, bazo, riñón e hígado y otras neoplasias intraabdominales y, especialmente, con otros quistes mesentéricos $^{4,8}$, por las características macroscópicas y microscópicas de la tumoración ${ }^{9}$. En el caso del linfangioma, los espacios quísticos están revestidos por una capa de endotelio y existen pequeños agregados linfoides en la pared del quiste ${ }^{4}$.

La presentación clínica es muy variable: no existe un patrón clínico constante debido a que los síntomas dependen de la presencia de la masa quística abdominal, de su localización, tamaño ${ }^{9} \mathrm{y}$, secundariamente, de las complicaciones que pueda provocar. Pueden ser asintomáticos durante años y la forma más frecuente de presentación es la de una masa blanda y palpable en abdomen, siendo el síntoma más frecuente el dolor abdominal ${ }^{3}$ y también las náuseas y/o vómitos. En niños es más común que puedan aparecer síntomas agudos debido a complicaciones ${ }^{5,7}$. Se han descrito casos de oclusión intestinal, peritonitis, infección, sangrado intraquístico, vólvulo intestinal o pueden confundirse con cuadros de apendicitis aguda o torsión de quiste anexial ${ }^{10}$.

El diagnóstico de sospecha se realiza por ultrasonografía y $\mathrm{TC}^{4}$ y la confirmación requiere una laparotomía seguida de un estudio anatomopatológico del tumor. La biopsia guiada preoperatoria no es útil debido a la dificultad de obtención de una muestra por la localización del tumor y a 
la posible diseminación tras la punción de la masa ${ }^{5}$.

El tratamiento de elección es quirúrgi$\operatorname{co}^{8}$ y debe intentarse una resección completa siempre que sea posible y no estén invadidas estructuras vitales. A menudo es preciso extirpar asas intestinales que comparten vascularización con el quiste o se ven atrapadas en él ${ }^{1,6}$, al igual que ocurrió en nuestra paciente, u otras estructuras comprometidas próximas a la lesión ${ }^{8}$. La marsupialización no da buenos resulta$\operatorname{dos}^{2}$. Se han publicado casos de excisión con éxito mediante laparoscopia ${ }^{11}$, incluso en quistes gigantes ${ }^{12} \mathrm{y}$ de tratamiento mediante sustancias esclerosantes ${ }^{13}$. El pronóstico de estas tumoraciones es excelente siempre que se logre la exéresis completa, ya que en caso contrario podría seguir un curso invasivo por proliferación tumoral. Se han descrito muy pocos casos de transformación maligna de quiste mesentérico ${ }^{9}$, como un adenocarcinoma metastásico a los 12 años de extirpar la lesión inicial ${ }^{14}$.

El seguimiento se realizará mediante ecografía abdominal para asegurar que la resección ha sido completa y descartar posibles recidivas posteriores ${ }^{9}$.

En conclusión, los linfangiomas quísticos han de ser incluidos en el diagnóstico diferencial de los diferentes quistes abdominales $\mathrm{y}$, aunque sean asintomáticos, su tratamiento debe ser quirúrgico con extirpación completa, debido a su potencial de crecimiento e invasión de estructuras vitales o al peligro de aparición de graves complicaciones ${ }^{10}$.

\section{BIBLIOGRAFÍA}

1. TAN JJ, TAN KK, CHEW SP. Mesenteric cysts: an institution experience over 14 years and review of literature. World J Surg 2009; 33: 1961-1965.
2. O'Brien MF, Winter DC, Lee G, Fitgerald EJ, O'Sullivan GC. Mesenteric cysts: a series of six cases with a review of the literature. Ir J Med Sc 1999; 168: 233-236.

3. Rivas ML, Asensio JA, Forno W, Petrone P, RolDÁN G, GaRcía W. Quistes mesentéricos. Cir Gen 2002; 24: 61-65.

4. Losanoff JE, Richman BW, El-Sherif A, Rider KD, JonEs JW. Mesenteric cystic lymphangioma. J Am Coll Surg 2003; 196: 598-603.

5. Cherk M, Nikfarjam M, Christophi C. Retroperitoneal lymphangioma. Asian J Surg 2006; 29: 51-54.

6. Luo CC, Huan CS, Chao HC, Chu SM, Hsueh C. Intra-abdominal cystic lymphangiomas in infancy and childhood. Chang Gung Med J 2004; 27: 509-514.

7. VALDIVIA GG. Quiste de mesenterio. Revisión. Rev Gastroenterol Mex 2003; 68: 235-238.

8. Weeda VB, BoojI KA, Aronson DC. Mesenteric cystic lymphangioma: a congenital and an acquired anomaly? Two cases and a review of the literature. J Pediatr Surg 2008; 43: 1206-1208.

9. De Perrot M, Bründler MA, Tötsch M, Mentha G, Morel P. Mesenteric cysts. Toward less confusion? Dig Surg 2000; 17: 323-328.

10. Losanoff JE, KJossev KT. Mesenteric cystic lymphangioma: unusual cause of intra-abdominal catastrophe in an adult. Int J Clin Pract 2005; 59: 986-987.

11. Dequanter D, Lefebvre JC, Belva P, Takieddine M, VANEUKEM P. Mesenteric cysts. A case treated by laparoscopy and a review of the literature. Surg Endosc 2002; 16: 1493.

12. Talarico F, Iusco D, Negri L, Valieri L. Mesenteric cystic lymphangioma treated with laparoscopic excision: case report and review of the literature. G Chir 2009; 30: 362-364.

13. Cabrera J, Redondo P. Tratamiento esclerosante de las malformaciones vasculares. An Sist Sanit Navar 2004; 27: 117-126.

14. Solis C, Mahmoud A, Rassai H. Retroperitoneal mesenteric duplication cysts with malignant transformation in adulthood. Am Surg 2009; 75: 261-263. 